\title{
Cerebellar Syndromes: A Medical Student Guide
}

Claudia K. Sellers', Suvankar Pal².

\begin{abstract}
.
The cerebellum is central to normal motor function and co-ordination, and can be frequently affected in a number of common disease processes. However, medical student teaching relating to cerebellar anatomy and pathology is lacking, leaving many graduates with a significant knowledge gap. Junior doctors need to be able to recognize 'cerebellar syndromes' on presentation to hospitals, and to identify and manage reversible causes rapidly and effectively. After review of relevant literature, a simple approach to the functional anatomy and practical classifications of common cerebellar pathology is presented here, with a focus on symptoms, signs and examination techniques essential to medical school final exams.
\end{abstract}

Keywords: Cerebellar Diseases; Central Nervous System Diseases; Cerebellum; Students, Medical (Source: MeSH, NLM)

\section{Introduction.}

The cerebellum (Latin for 'little brain') is located infero-posteriorly to the cerebral cortex, and is fundamental to normal neurological functioning, yet it was not until the early 2oth Century that Flourens discovered its primary function to be in motor control and co-ordination.' The cerebellum may be affected in common neurological disorders such as stroke, multiple sclerosis and mass lesions, often producing a 'cerebellar syndrome'. Junior doctors need to be confident in recognition and investigation of patients presenting with cerebellar symptoms and signs appropriately in order to identify treatable causes and provide accurate prognosis. Textbook coverage of this area is notoriously disjointed, often leaving medical students with a significant knowledge gap. This article aims to provide a brief overview of the etiology of cerebellar disorders and a simple approach to history, examination and investigation of patients presenting with cerebellar syndromes.

\section{Methodology.}

A review of literature was performed using PubMed, Google Scholar, Embase and Medline databases for articles from 1975 to 2012 using the search terms: Cerebellum, Neurological Examination, Ataxia, and Cerebellar Syndromes. Additional papers were retrieved from reviews and references. Articles included were in the English language and related to anatomy and clinical examination technique of the cerebellar system.

\section{Anatomy.}

The cerebellum is attached to the dorsal aspect of the brainstem by three separate fibrous structures - the superior, middle and inferior peduncles, which connect to the mid brain, pons and medulla respectively (Figure 1). It comprises two hemispheres joined in the midline by the 'vermis', which, unlike the cerebral hemispheres, are ipsilateral in their control of co-ordination. Afferent input to the cerebellum includes neuronal tracts from the semicircular canals, proprioception via the spinal cord, motor information from the frontal lobes, and fibers from reticular nuclei in the brainstem. Efferent output is to midbrain and thalamic nuclei, or directly to the motor cortex, permitting feedback comparing sensory and motor input with output.

The anatomy of the cerebellum can also be further divided functionally. Control of balance, posture and eye movements is mediated by the flocculonodular lobe (or archicerebellum) which connects with the vestibular and reticular nuclei in the brainstem. The vermis and paravermis (the intermediate section between the cerebellar hemispheres) together make up the paleocerebellum, responsible for control of postural and distal muscle tone. Finally, coordination and planning of limb movement occurs within the neocerebellum, which is comprised of the remaining cerebella hemispheres. ${ }^{2}$

\section{Etiology of Cerebellar Disease}

Cerebellar pathology arises secondary to a broad range of etiologies which can be usefully classified according to the time-course of symptoms:

1. Acute onset (seconds to minutes)

Usually cerebellar infarction or hemorrhage. This may be
Abbreviations Index: CID, Creutzfeld-Jacob Disease CSF, Cerebrospinal Fluid CT, Computed Tomography CXR, Chest X-ray $\mathrm{GI}$, Gastrointestinal HIV, Human Immunodeficiency Virus

MRI, Magnetic Resonance Imaging OSCE, Objective Structured Clinical Examination TIA, Transient Ischemic Attack

About the author.

Claudia Sellers is a Foundation Year 1 Doctor at Guy's and St Thomas' NHS Trust, London, UK, and graduated from the University of Edinburgh Medical School in 2011.

\footnotetext{
${ }^{1}$ Department of Acute General Medicine, St Thomas' Hospital, London, UK.

${ }^{2}$ Department of Clinical Neurosciences, Western General Hospital, Edinburgh, Scotland.

Correspondence

Dr. Claudia Sellers.

Address: Department of Acute General Medicine, St Thomas' Hospital, London SE1 7EH.

E. mail: claudiesellers@hotmail.co.uk
} 
associated with headache, vertigo, vomiting and altered consciousness.

2. Sub-acute ataxias (hours to days)

- Inflammatory causes - most commonly multiple sclerosis

- Viral - (more common in children) associated with pyrexia, dysarthria, limb at gait ataxia.

- Paraneoplastic cerebellar degeneration. Particularly carcinomas of ovaries and lung.

- Other - hydrocephalus, posterior fossa tumors, abscesses, parasitic infections, toxins.

- Alcohol excess

\section{Episodic ataxias}

Usually transient and last minutes to hours, there are various causes:

- Drugs: Barbiturates, Phenytoin and other Anticonvulsants, Anti-neoplastic drugs
- Transient posterior cerebral circulation (vertebrobasilar) ischemic attacks

- Foramen magnum compression

- Inherited episodic ataxias

4. Chronic progressive ataxias (months to years)

- Chronic alcohol excess

- Malnutrition (including thiamine, zinc and vitamin E deficiencies).

- Drugs - particularly anticonvulsants such as phenytoin (may reverse once withdrawn)

- Structural lesions (slowly progressive tumors or vascular malformations)

- Inherited: Including Friedreich's ataxia, spinocerebellar ataxias

- Degenerative: Including multiple system atrophy, idiopathic late onset ataxias

- Rarer causes: Chronic solvent abuse, heavy metal exposure, prion diseases (including sporadic CJD)

Figure 1. Anatomy of the Cerebellum.

Archicerebellum:

Control of balance and eye movements.

ANTERIOR VIEW

Afferent input to cerebellum includes:

- Semicircular canals.

- Spinal cord.

\section{SUPERIOR \\ VIEW}

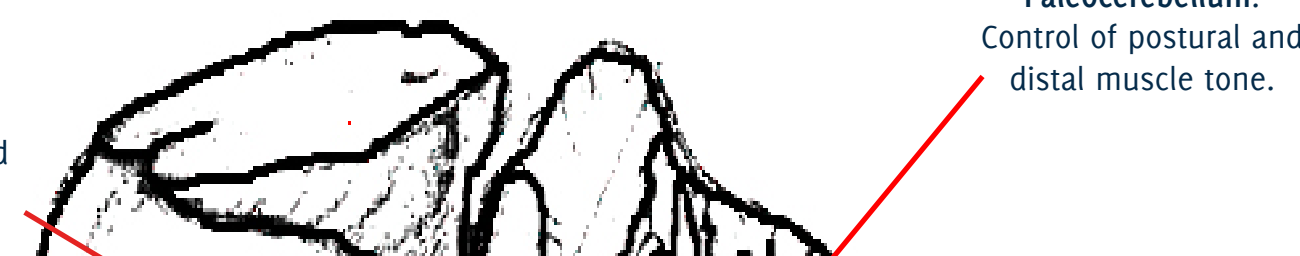

POSTERIOR VIEW

Neocerebellum:

Coordination and planning

of limb movement. 
A 'surgical sieve' approach to the commoner causes is illustrated in Table 1.

\section{Symptoms and signs of cerebellar disease}

The cerebellum operates at a subconscious level to control muscle tone, posture and co-ordination. Cerebellar disorders result in difficulties with the rate, rhythm and force of limb movements, gait and speech. ${ }^{3}$ The key symptoms and signs can be remembered using the mnemonic DANISH:

- Dysdiadochokinesis - Inability to perform rapid alternating movements, especially of limbs.

- Ataxia - Inco-ordination of voluntary movements. This includes 'dysmetria': inability to accurately judge distance, leading to over- or under-shooting of targets and unsteady gait.

- Nystagmus - Rapid, involuntary movement of the eyes.

- Intention tremor - Tremor exacerbated by voluntary goal-directed movements.

- Scanning dysarthria - Jerky, sometimes explosive, slurred speech, with difficulties maintaining rate, rhythm and force resulting in separated syllables.

- Heel-shin ataxia.

Patients may also present with symptoms relating to underlying pathology for example, headache, nausea and vomiting resulting from a cerebellar tumor; systemic upset associated with a cerebellar abscess, and pyramidal and sensory signs in multiple sclerosis.
Figure 2. CT head scan showing right sided cerebellar hemorrhage. This elderly patient presented with sudden onset headache, nausea, vomiting, unsteady gait and right sided clumsiness.

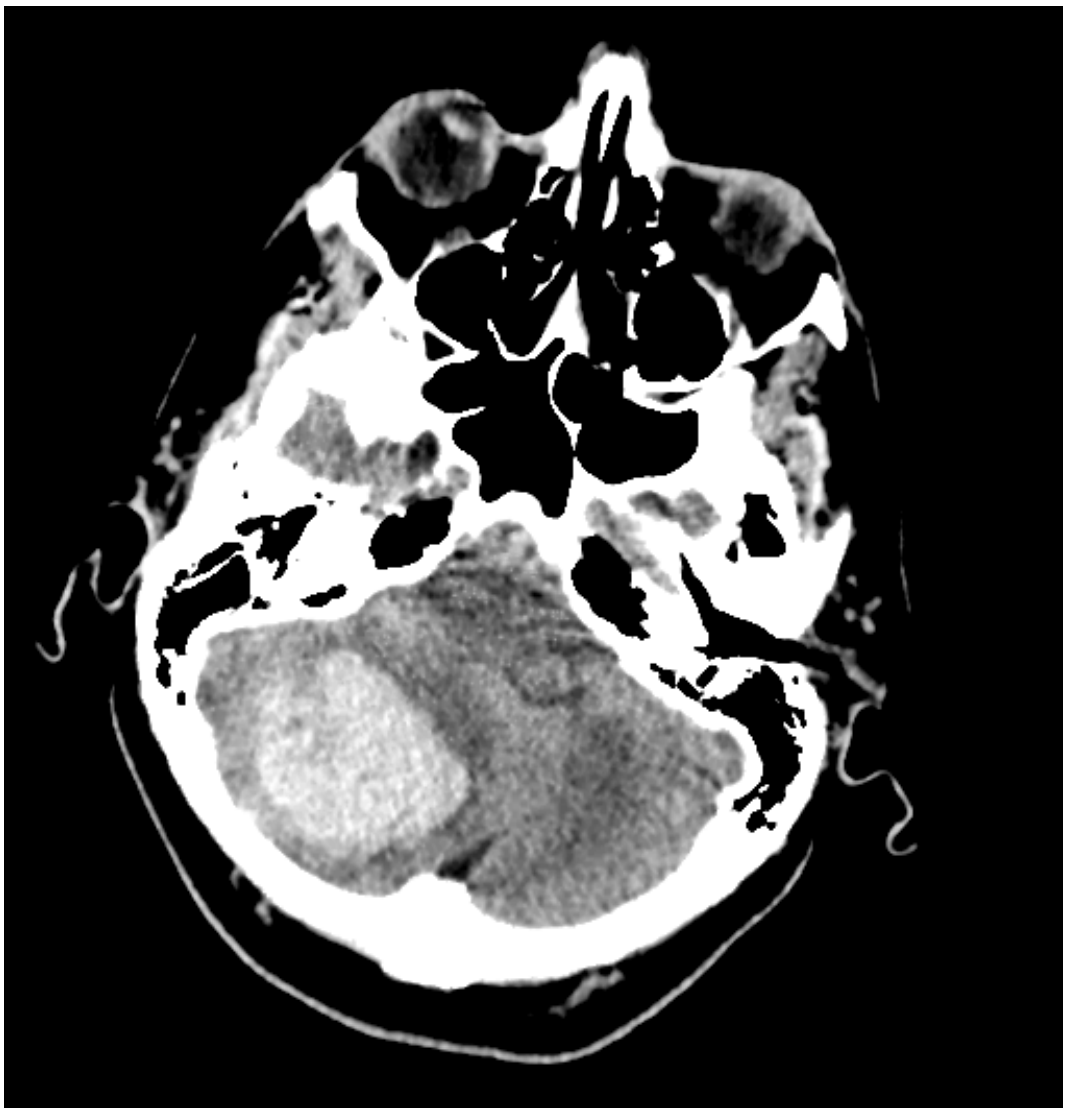

Figure 3. A (axial section) and B (sagittal section). MRI head scan showing mixed solid/cystic left cerebellar hemisphere mass with hydrocephalus and cerebellar tonsillar herniation caused by a pylocytic astrocytoma. This young patient presented with a subacute left sided cerebellar syndrome associated with headache.
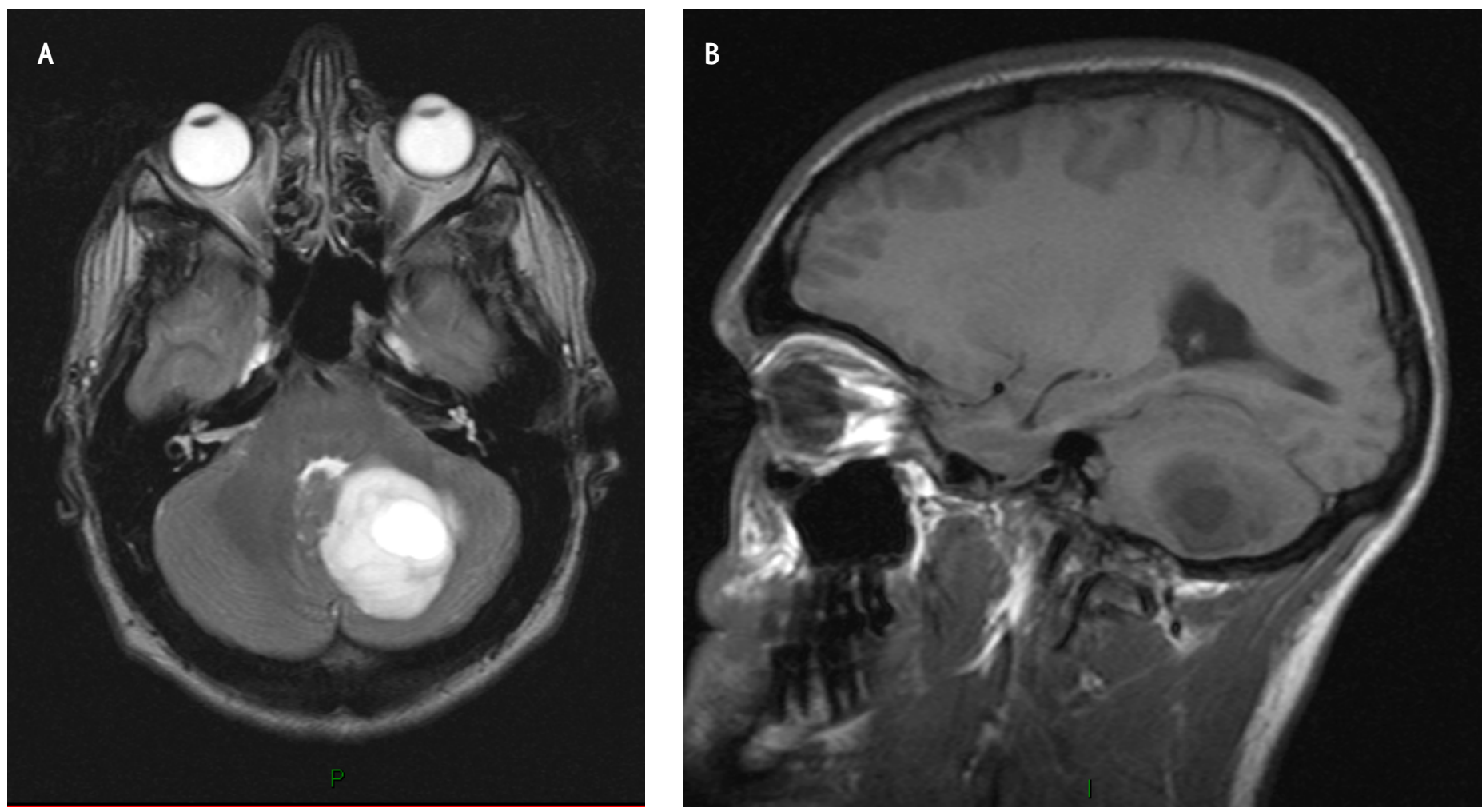
A guide to comprehensive examination of cerebellar function as may be expected in an undergraduate objective Structured Clinical Examination (OSCE) is presented in Table 2.

Handy Hints:

1. Romberg's sign is often mistakenly attributed to cerebellar dysfunction. It is actually primarily a sign of pathology affecting the dorsal columns. ${ }^{4}$ The patient stands with their feet together and eyes closed; if they lose balance, the test is positive. Postural hypotension, anxiety and axial stiffness are potential causes of a false positive Romberg's. In cerebellar ataxia, the test is not positive: the patient cannot balance with eyes open

2. Note that rapid repetitive movements can also be used to detect non-cerebellar problems; for example, in upper motor neuron lesions these movements are slow and deliberate, and in extrapyramidal conditions such as Parkinson's the movements start slowly, and may be small in amplitude and slow in speed.

3. In disorders which predominantly affect midline cerebellar structures (tumors of the vermis/alcohol-induced damage), the finger-nose test, heel-shin test and testing for dysdiadochokinesis may be normal. It is therefore of important to check the stance and gait in cerebellar examination, as truncal ataxia may be the only abnormal finding.

"Overall, specific tests can be helpful in confirming or refuting the presence of cerebellar disease, but must be interpreted in the context of other signs and the case history."

\section{Investigation of Cerebellar Disease}

Investigation should be guided according to the differential diagnoses in mind, but commonly includes blood tests and cerebellar imaging.

\section{An approach to investigating cerebellar disorders:}

- Toxic: Review drug charts, interactions, toxicology screen.

- Infectious: Temperature, pulse and blood pressure, full blood count, blood cultures.

- Metabolic/endocrine: Thyroid function, caeruloplasmin, copper, thiamine, blood glucose.

- Inflammatory (including multiple sclerosis): CSF examination for white cell count, protein and oligoclonal bands.

- Neoplastic/Paraneoplastic: CXR, body imaging, tumor markers, paraneoplastic antibodies.

- Genetic: Blood testing for genetic mutations - Friedrich's ataxia, spinocerebellar ataxias.

Imaging: Computed tomography (CT) (Figure 2) or Magnetic resonance tomography (MRI) (Figure 3 ) of the brain are used to investigate cerebellar disorders and may reveal a stroke, space occupying lesion, demyelination or atrophy.

\section{In Summary.}

The cerebellum is affected by a number of common disease processes, producing characteristic symptoms and signs. Junior doctors need to be able to accurately detect such 'cerebellar syndromes' since they are a common presentation to hospital and since there are a number of treatable causes which can be reversed if detected early. ${ }^{5} \mathrm{~A}$ sound understanding of the anatomy of the cerebellum, etiology of cerebellar disorders and logical approach to examination will facilitate appropriate investigation and treatment. ${ }^{6}$

- Causes of cerebellar disease: Commonly stroke/TIA; multiple sclerosis; mass lesions including primary tumors, metastases and abscesses; drugs; alcohol and metabolic abnormalities.

- Symptoms of cerebellar disease: Difficulties with coordination, intention tremor, gait disturbance, slurred speech.

Table 1. 'Surgical Sieve' approach to common causes of cerebellar syndromes. * Denotes common cause.

\begin{tabular}{ll}
\hline Vascular & Stroke (infarct or hemorrhage)*, Transient ischemic attack* \\
Inflammatory & Multiple sclerosis* \\
\hline Neoplastic & Primary tumors: Astrocytoma, Medulloblastoma, Haemangioblastoma \\
& Secondary tumors: metastases* (commonly lung, breast and Gl tract) \\
& Paraneoplastic phenomena (Anti Hu Ab in small cell lung cancer) (rare) \\
\hline Toxic/Trauma & Alcohol* $^{*}$ \\
\hline Metabolic & Hypoglycemia*, Hypoxia*, Hypothyroidism*, thiamine deficiency* \\
\hline Infectious & Bacterial: Meningo-encephalitis, Intracranial abscess, Viral: Varicella, HIV \\
\hline Congenital & Parasitic infections(rare): Toxoplasma, Falciparum Malaria \\
\hline Inherited & Agenesis, Dandy-Walker malformation, Arnold-Chiari malformation (rare) \\
\hline Degenerative & Friedrich's Ataxia, spinocerebellar ataxias \\
Drugs & Multisystem atrophy, Spinocerebellar ataxias, prion disease (rare) \\
\hline
\end{tabular}


Table 2. A systematic approach to cerebellar examination. 'Please examine this patient's cerebellar function'. How to pass the OSCE.

\begin{tabular}{|c|c|c|}
\hline & Action & Potential Findings \\
\hline Introduction & $\begin{array}{l}\text { Wash your hands. } \\
\text { Introduce, explain, consent the patient. } \\
\text { Position the patient at } 45^{\circ} \text { lying down. }\end{array}$ & \\
\hline Inspection & $\begin{array}{l}\text { Bruising, scars } \\
\text { ‘bobbing ' head } \\
\text { Walking aids }\end{array}$ & $\begin{array}{l}\text { Recurrent falls } \\
\text { Titubation } \\
\text { gait ataxia }\end{array}$ \\
\hline
\end{tabular}

\begin{tabular}{|c|c|c|}
\hline \multicolumn{3}{|l|}{ Head } \\
\hline Nystagmus & Follow your finger with eyes, head still & $\begin{array}{l}\text { Broken pursuit, nystagmus the degree of involuntary eye } \\
\text { movement is greatest when gaze is focused to the same side } \\
\text { as the lesion. }\end{array}$ \\
\hline Speech & $\begin{array}{l}\text { Ask to repeat 'West Register Street, British Constitution, PP PP } \\
\text { PP, KK KK KK, TT TT TT' } \\
\text { Ask to read a sentence aloud }\end{array}$ & $\begin{array}{c}\text { Staccato Speech } \\
\text { Slurring }\end{array}$ \\
\hline
\end{tabular}

\section{Limbs}

Tone

As in normal neurological exam

Power

As in normal neurological exam

May be increased after a stroke or in multiple sclerosis.

Coordination

Test for 'rebound':

-Arms out straight in front with eyes closed,

push each arm down $\sim 10 \mathrm{~cm}$ then release6

\section{Finger-nose Test}

Examiner holds index finger about $50 \mathrm{~cm}$ in front of the patient's face, and asks the patient to go between touching his/her nose and the examiner's finger as accurately as possible.

Hand-turning test

Ask the patient to tap one hand on the back of the other, and

then tap the same spot with the dorsum of the same hand.

Coordination

\section{LOWER LIMBS}

Heel-shin test

Put heel onto opposite knee and slide heel

down shin to ankle. Lift off and repeat.

Foot tapping

Ask to tap foot as rapidly as possible

\section{Posture and Gait}

Posture

Ask how stable they are sitting/standing?

Assess sitting down

Also with arms crossed

Assess standing (If stable sitting)

Arms by sides, feet together

Ask to walk across room

Wide-based gait (midline cerebellar lesions)

Ask to walk heel to toe Unsteadiness and lateral veering (hemispheric lesions) Irregular steps
Difficulty $=$ Dysmetria Intention tremor, past pointing

Difficulty $=$ Dysdiadochokinesis gy. $\downarrow$ power may confound incoordination

Concluding Remarks

"I would also like to carry out a full neurological examination"

Investigations: MRI posterior fossa, and specific as indicated

(see text)

- Signs of cerebellar disease: Dysdiadochokinesis a Dysmetria, Ataxic gait, Nystagmus, Intention tremor, Scanning dysarthria, Heel-shin test ataxia (remember DANISH).

Investigations: Blood tests, Imaging, CSF examination and genetic screening as appropriate. 


\section{References.}

1. Fine EJ, Ionita CC, Lohr L. The history of the development of the cerebellar examination. Semin Neurol 2002; 22(4): 375-84

2. Levy MN, Koeppen BM, Stanton BA. Special Senses: The cerebellum assists in the regulation of posture and movement. In: Levy MN, Koeppen BM, Stanton BA (4th ed). Principles of Physiology. Philadelphia: Elsevier Mosby; 2006.

3. Shardlow A, Turner MR. Examination of the Cerebellum. J Clin Exam 2008; 5:5-9.

4. Khasnis A, Cokula RM. Romberg's test. J Postgrad Med 2003; 49(2):169-72.

5. Manto M. The cerebellum, cerebellar disease and cerebellar researchtwo centuries of discoveries. Cerebellum 2008;7(4):505-16.

6. Angel RW. The rebound phenomenon of Gordon Holmes. Arch Neurol $1977 ; 34(4): 250$. 